\title{
Performance Evaluation of a DS-CDMA System in a Rayleigh Fading Environment
}

\author{
M. A. Rahman1, M. M. Alam1, Md. Khalid Hossain 2*, Md. Khairul Islam¹, \\ Khan M. Nasir Uddin', Md. Shahinuzzaman'1 \\ ${ }^{1}$ Department of Applied Physics, Electronics \& Communication Engineering, Islamic University, \\ Kushtia, Bangladesh \\ ${ }^{2}$ Institute of Electronics, Atomic Energy Research Establishment, Savar, Bangladesh \\ Email: *khalid.baec@yahoo.com
}

Received 1 November 2015; accepted 19 December 2015; published 23 December 2015

Copyright @ 2016 by authors and Scientific Research Publishing Inc.

This work is licensed under the Creative Commons Attribution International License (CC BY). http://creativecommons.org/licenses/by/4.0/

c) (i) Open Access

\section{Abstract}

This work represents the performance evaluation of DS-CDMA in a Rayleigh fading environment. Performance analysis was carried out for a DS-CDMA system to evaluate the signal-to-noise ratio (SNR), signal-to-interference ratio (SIR) and bit error rate (BER) for different modulation schemes like ASK, PSK and FSK. Results were evaluated at different data rates and were presented in terms of SNR, SIR and BER. Computer simulation was also carried out by using Matlab to evaluate the signal waveforms with and without addition white Gaussian noise (AWGN). Butterworth filtered waveform was given to get better signal quality. Decoded output waveforms were also evaluated to confirm the simulation.

\section{Keywords}

DS-CDMA, Rayleigh Fading, Performance Analysis, SNR, BER, ASK, PSK, FSK, MATLAB, AWGN

\section{Introduction}

In digital communication system, selection of the most appropriate access method is a challenging task. To meet this challenge we have to be familiar with the technologies and system architectures of the CDMA digital cellular system. CDMA development started in early 1989 after the NA-TDMA standard was established. In early 1990, QUALCOM incorporated of San Diego, California pioneered to introduce the intensive system concepts [1] and the innovative implementation approaches on CDMA channel structure, interference, gain, etc. In the recent years, Code Division Multiple Access (CDMA) draws considerable interest of the wireless communication engineers to be used as a multiple access technique because of its some invaluable advantages [2] over Time Division Multiple Access (TDMA) and Frequency Division Multiple Access (FDMA).

How to cite this paper: Rahman, M.A., Alam, M.M., Hossain, M.K., Islam, M.K., Uddin, K.M.N. and Shahinuzzaman, M. (2016) Performance Evaluation of a DS-CDMA System in a Rayleigh Fading Environment. World Journal of Engineering and Technology, 4, 1-9. http://dx.doi.org/10.4236/wjet.2016.41001 
The tremendous demand from the subscribers of mobile communication leads to the development of new standards for this technology. Various application areas are evolving in this field. To meet the future demands, the ITU, IMT-2000 plan was formulated [3]. IMT-2000 has been successful in helping to cultivate active debate and technical analysis for new high speed mobile telephone solutions when compared to 2G. The eventual 3G evolution for CDMA systems leads to CDMA2000. Several variants of CDMA2000 are currently being developed, but they all are based on the fundamentals of IS-95 and IS-95B technologies. The eventual 3G evolution for GSM, IS-136, and PDC systems leads to wideband CDMA (W-CDMA), also called Universal Mobile Telecommunications Service (UMTS). W-CDMA is based on the network fundamentals of GSM, as well as the merged versions of GSM and IS-136 through EDGE. It is fair to say that these two major 3G technology camps, CDMA2000 and W-CDMA, will remain popular throughout the early part of the 21st century. Since 1998, many standards proposals [4]-[8] have come out and joined with either the CDMA2000 or UMTS (W-CDMA) camps. The ITU IMT-2000 standards organizations are currently separated into two major organizations reflecting the two 3 G camps.

In the recent years, wireless CDMA draws considerable interest of the communication engineers to be used as a technique for multiple access communications. CDMA has many advantages over the other traditional multiple access techniques such as TDMA and FDMA. CDMA can accommodate more users than TDMA and FDMA, interference rejection, suppression and multi-path mitigation are better in CDMA than TDMA and FDMA [9]-[12].

Direct sequence CDMA (DS-CDMA) and frequency hopping CDMA (FH-CDMA) are the two types of CDMA. DS-CDMA is the most popular between them for its simplicity, better performance and more reliability. Many research works have been done [13] on the performance of reverse link and forward link of DS-CDMA mobile communication system. DS-CDMA systems are generally considered to be uplink limited. Multiple Access Interference (MAI) is a factor which limits the capacity and degrades the performance of DS-CDMA systems. Any analysis of performance of a CDMA system has to take into account the amount of MAI and its effects on the parameters that measure the performance, especially Signal-Noise Ratio (SNR) and Bit Error Rate (BER).

The BER performance of DS-CDMA system in a Rayleigh fading channel has been investigated in this research. So far, most of the researches on DS-CDMA mobile communication systems were done considering the mobile wireless channel either AWGN channel or Rayleigh distributed flat or frequency selective fading channel. The objectives of the current research work were: 1) To study a Direct Sequence Code Division Multiple Access (DS-CDMA) system in a Rayleigh fading channel, 2) To carry out the analysis for carrier to multiple access interference ratio and the compression for bit error rate (BER) of a DS-CDMA system in a Rayleigh fading channel, 3) To carry out numerical computations to evaluate the carrier to interference ratio (CIR) and to find the BER as a function of system parameters, 4) To carry out computer simulations to evaluate the signal waveforms and the amount of signal distortions due to fading and 5) To find the optimum system parameters in presence of fading.

\section{DS-CDMA}

In DS-CDMA is the most popular of the CDMA techniques. A DS-CDMA signal can be achieved when the original narrowband signal $b(t)$ is directly multiplied by a wideband signal $a(t)$ resulting in the signal $b(t) a(t)$ as shown in Figure 1. This wideband signal consists of a sequence of small Pseudo-Noise (PN) chips whose interval Tc is much smaller than the symbol interval Ts. The PN sequence is called code sequence or spreading sequence. The multiplication of data signal by the PN sequence is called spreading. The ratio Ts/Tc $=\mathrm{Gp}$ is called the processing gain. The spread spectrum signal can be converted (de-spread) back to the original signal by simply multiplying the received sequence with the same spreading sequence.

As mentioned earlier, in the DS-CDMA systems the modulated information bearing signal (the data signal) is directly modulated by a digital code signal. The data signal can be either an analog signal or a digital one. In most cases it will be a digital signal. What we often see in the case of a digital is that the data modulation is omitted and the data signal is directly multiplied by the code signal and the resulting signal modulates the wideband carrier. It is from this multiplication that the direct- sequence CSMA get its name. In Figure 2 a block diagram of a DS-CDMA transmitter is presented. The binary data signal modulates an RF carrier. The modulated carrier is then modulated by the PN sequence. The chips of PN sequence. The chips of a PN sequence can be either +1 or -1 . 


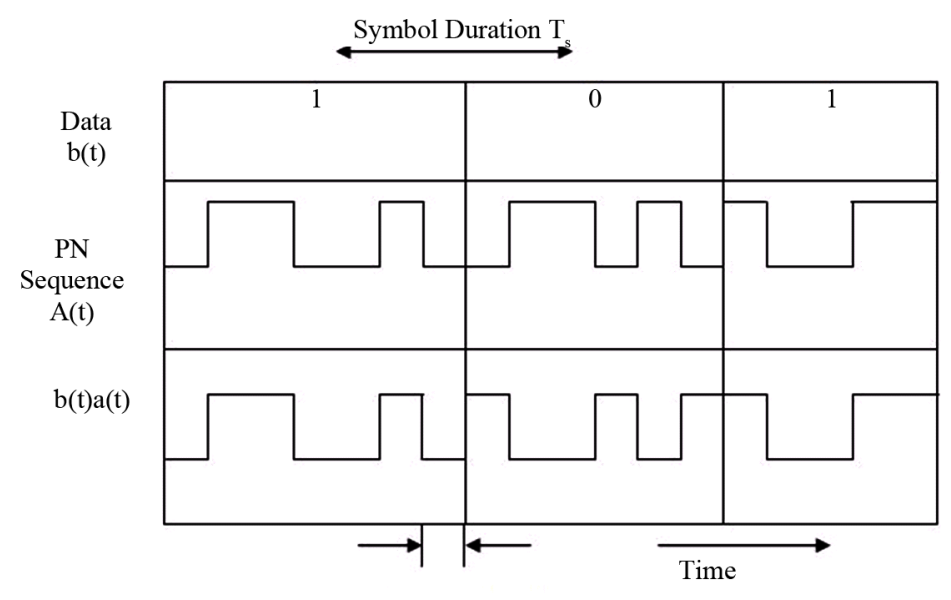

Chip Duration Tc

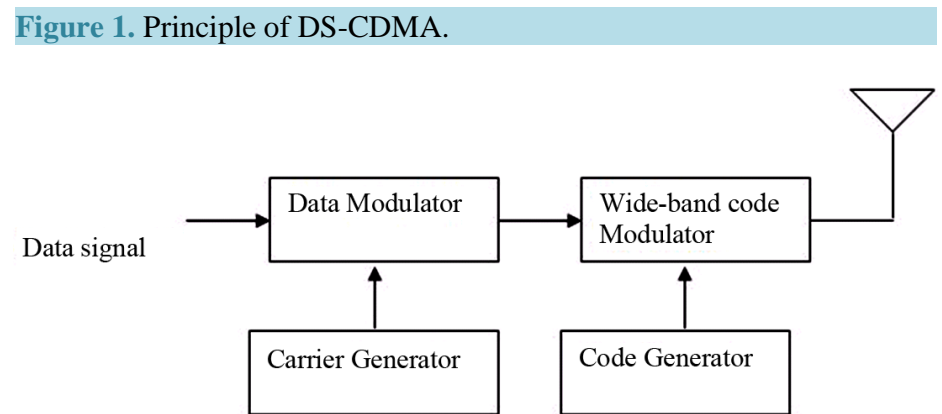

Figure 2. Bock diagram of a DS-CDMA transmitter.

To obtain the desired spreading of the signal, the chip rate of the PN sequence must be much higher than the chip rate of the information signal. After transmission of the signal, the receiver uses coherent demodulation to de-spread the spread spectrum (SS) signal, using a locally generated code sequence. To be able to perform the de-spreading operation, the receiver must not only know the code sequence used to spread the signal but also the codes of the received signal and the locally generated code must also be synchronized. This synchronization must be accomplished at the beginning of the reception and maintained until the whole signal has been received. After de-spreading the modulated data signal and after demodulation the original data can be recovered. Figure 3 represents the DS-CDMA receiver structure.

\section{Rayleigh Fading}

Fading is the term used to describe the rapid fluctuations in the amplitude of the received radio signal over the short period of time fading is a common phenomenon in mobile communication channels caused by the interference between two or more versions of the transmitted signals which arrive at the receiver at slightly different times. The resultant received signal can vary widely in amplitude and phase, depending on various factors such as the intensity, relative propagation time of the waves, bandwidth of the transmitted signal etc. the performance of a system can be severely degraded by fading. A typical mobile radio environment is shown in Figure 4. Due to the multi-path reflections from the various objects, the electromagnetic waves travel along different paths of varying length.

Rayleigh fading is a statistical model for the effect of a propagation environment on a radio signal, such as that used by wireless devices [14]. Rayleigh fading models assume that the magnitude of a signal that has passed through such a transmission medium (also called a communications channel) will vary randomly, or fade, according to a Rayleigh distribution - the radial component of the sum of two uncorrelated Gaussian random variables. Rayleigh fading is viewed as a reasonable model for tropospheric and ionospheric signal propagation as well as the effect of heavily built-up urban environments on radio signals [15] [16]. Rayleigh fading is most applicable when there is no dominant propagation along a line of sight between the transmitter and receiver. If 


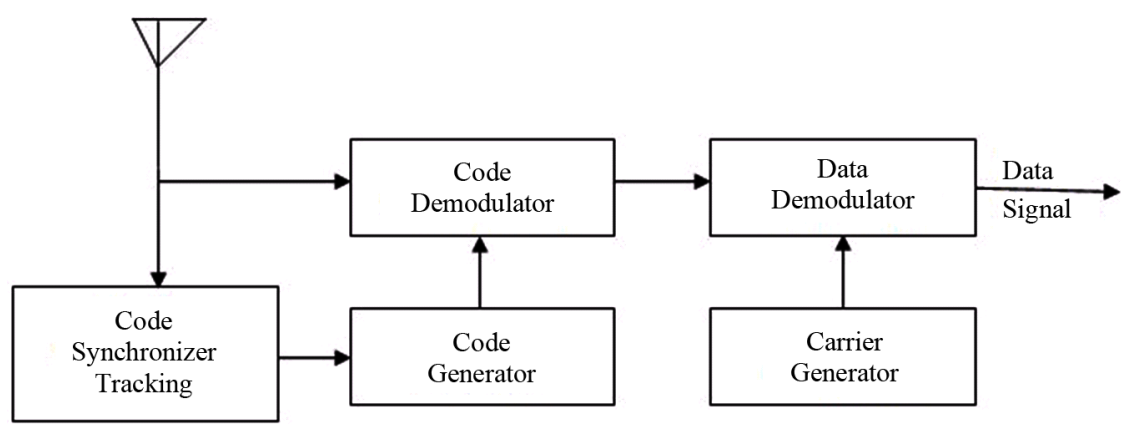

Figure 3. Block diagram of a DS-CDMA receiver structure.

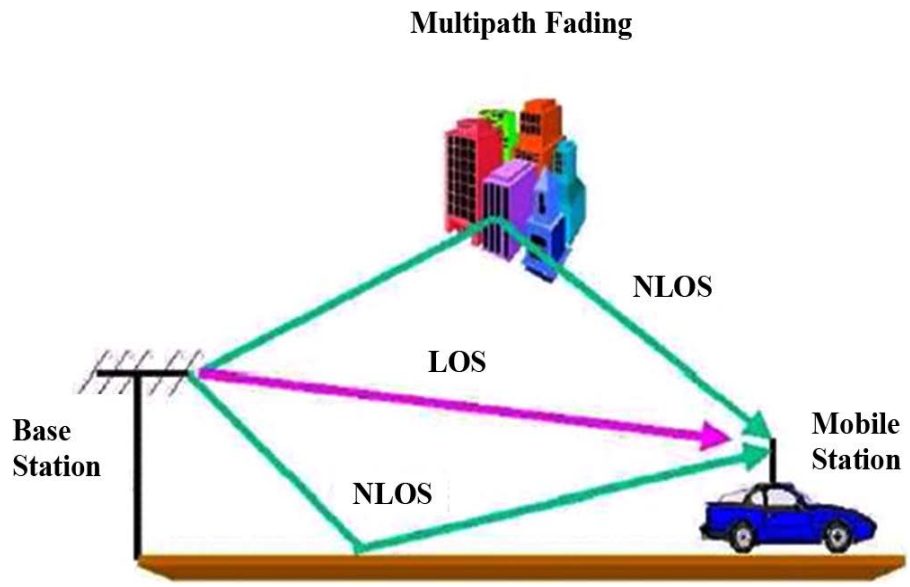

Figure 4. A Mobile Radio Environment Multi-path Fading.

there is a dominant line of sight, Rician fading may be more applicable [14].

\section{Results and Discussion}

The performance of DS-CDMA wireless cellular and non cellular (single cell) mobile communication system in a Rayleigh fading environment has been analyzed in this research work. The numerical results were evaluated considering the near far problem. The performance results were evaluated in terms of Signal-to-Noise Ratio (SNR), Signal-to-Interference Ratio (SIR) and the Bit Error Rate (BER) of the DS-CDMA system.

Here Matlab programs have been used to get various graphs. Analytical approach of a wireless communication system was followed considering Rayleish fading channel. The results were represented in terms of Signal-to-Noise ratio (SNR) and the Bit Error Rate (BER) for practical values of system parameters. The plot of SNR versus received power at the receiver input, $\operatorname{Pr}(\mathrm{dbm})$ is shown in Figure 5(a). It was found that the SNR increases with increase in the values $\operatorname{Pr}(\mathrm{dbm})$. Figure 5(b) represents the plots of SNR Vs Pr (dbm) for several values of data rates as $10 \mathrm{~kb} / \mathrm{s}, 15 \mathrm{~kb} / \mathrm{s}, 20 \mathrm{~kb} / \mathrm{s}, 30 \mathrm{~kb} / \mathrm{s}$, etc. From the graph we observed that at higher values of data rate the SNR were low which was due to higher noise power at the receiver output caused by higher filter bandwidth. From Figure 5(c) we observed that with the increase of transmitted signal power SNR increases Figure 5(d) shows the relation between distances vs. received power. From Figure 5(d) we observed that with the increase of distance the received power decreases due to higher path loss.

Bit error rate (BER) is the number of erroneous bits divided by the total number of bits transmitted received or processed over some stipulated period. Example of bit error ratio are a) transmission BER, that is the number of erroneous bits received divided by the total number of bits transmitted and b) information BER, i.e. the number of erroneous decoded (corrected) bits divided by the total number of decoded (corrected) bits. The BER is usually expressed as a coefficient and a power of 10; for example, 2.5 erroneous bits out of 100,000 bits transmitted would be 2.5 out of 105 or $2.5 \times 10^{-5}$. The Bit Error Rate (BER) of a communications system is a measure of the ratio between the number of bit errors for an estimated received signal with respect to the transmitted 


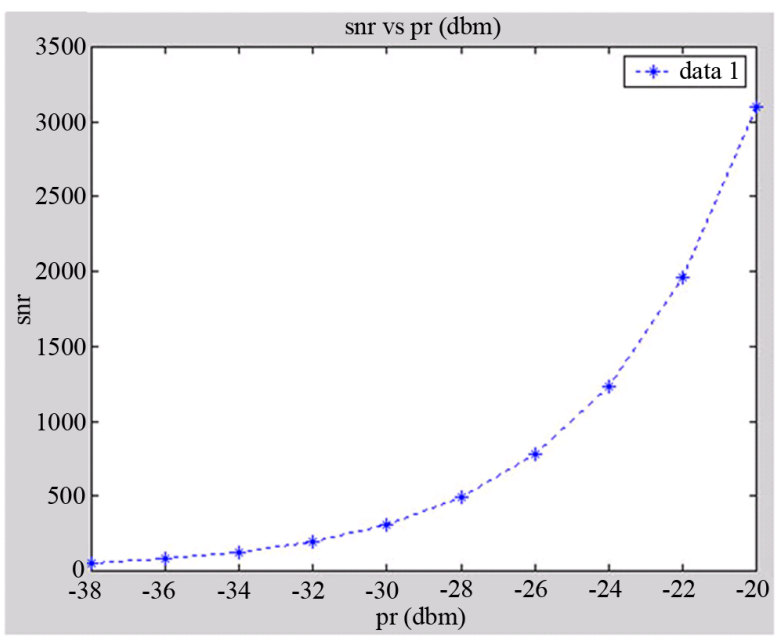

(a)

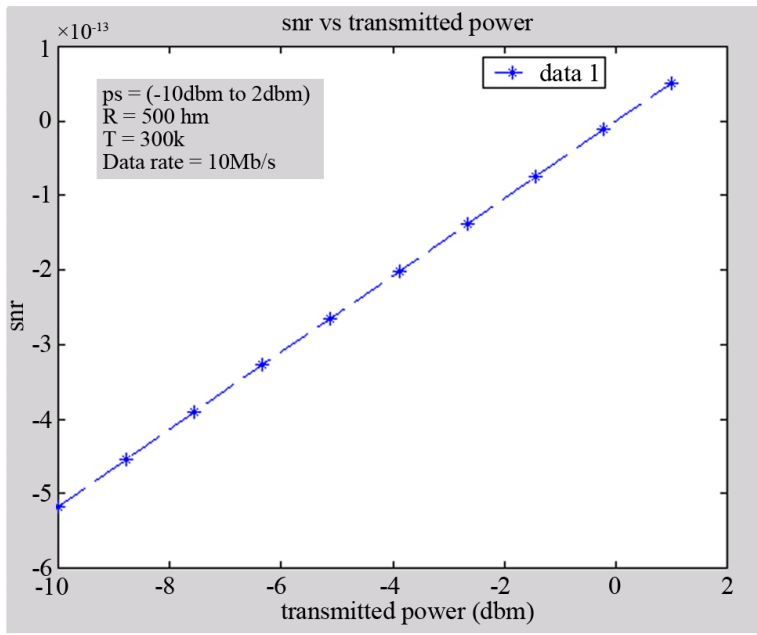

(c)

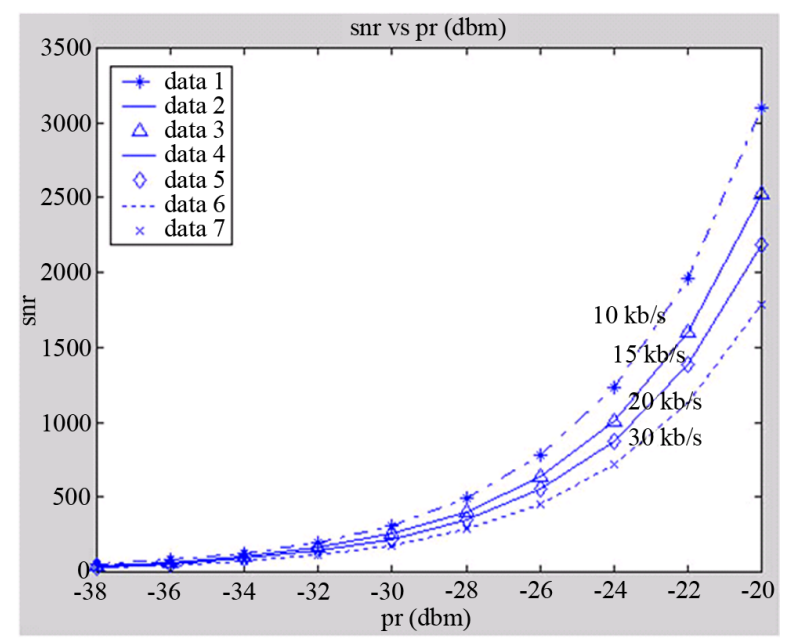

(b)

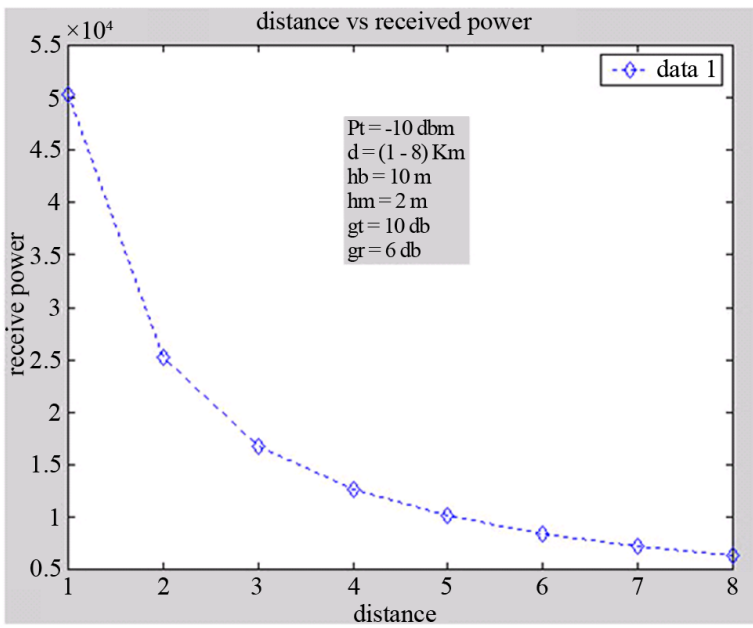

(d)

Figure 5. (a) Plot of SNR Vs Pr (dbm) at a bit rate $10 \mathrm{~kb} / \mathrm{s}$ for Rx, (b) plot of SNR Vs Pr(dbm) at different bit rate which are $10 \mathrm{~kb} / \mathrm{s}$ indicated by “*”, $15 \mathrm{~kb} / \mathrm{s}$ indicated by “ $\wedge$ ”, $20 \mathrm{~kb} / \mathrm{s}$ indicated by diamond and $30 \mathrm{~kb} / \mathrm{s}$ indicated by “x”, (c) relation between SNR Vs transmitted power. and (d) distance vs. received power.

signal and the total number of bits transmitted. Therefore the lower the bit error is, the better the system performance is. Typical communications systems use BER as the primary metric in evaluating the system performance. The BER is evaluated in term of 1) BER Vs SNR (db) and 2) BER Vs No of user.

Figure 6(a) represents the plots of BER vs. SNR for different data rates. From Figure 6(a) it was observed that with the increase of SNR the BER decreases. The plot shows the relation for ASK, PSK and FSK. All of them PSK is better because it has lower BER at high SNR then FSK and ASK. ASK has higher BER performance. Figure 6(b) shows the relation between the No. of user vs. power. From Figure 6(b) we observed that if we want to increase the number of users we must increase the transmitter power to cope with the multi-access interference (MAI). Figure 6(c) shows the relation between SIR Vs No. of simultaneous Users in a DS-CDMA system. From Figure 6(c) we observed that the SIR decreases with the increase in the number of users due to increased values of MAI. Figure 6(d) shows the relation between BER Vs SIR. From Figure 6(d) we observed that the BER decreases with the increase of the SIR. A higher value of SIR is required to have a lower value of BER. Figure 6(e) shows the relation between BER vs. no. of simultaneous user in a DS-CDMA system. From Figure 6(e) we observed that the BER increases with the increase of the number of users. The graphs are also plotted for PSK, FSK and ASK modulated DS-CDMA system. It was noticed that at a given BER more number 


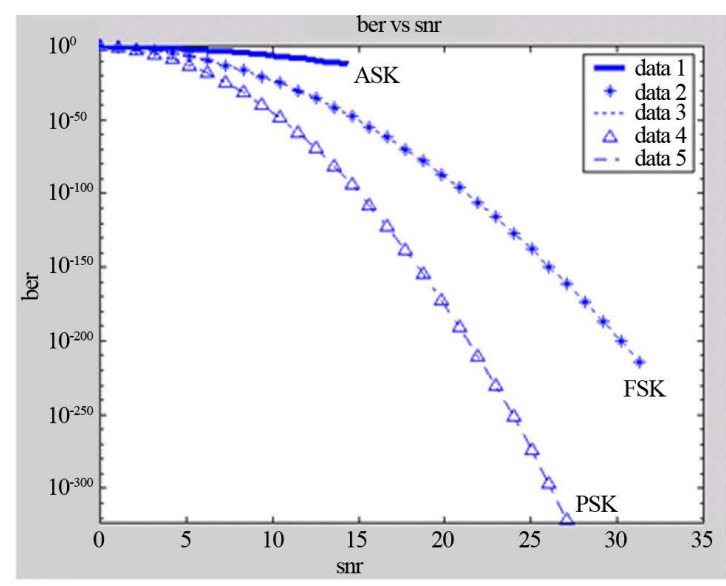

(a)

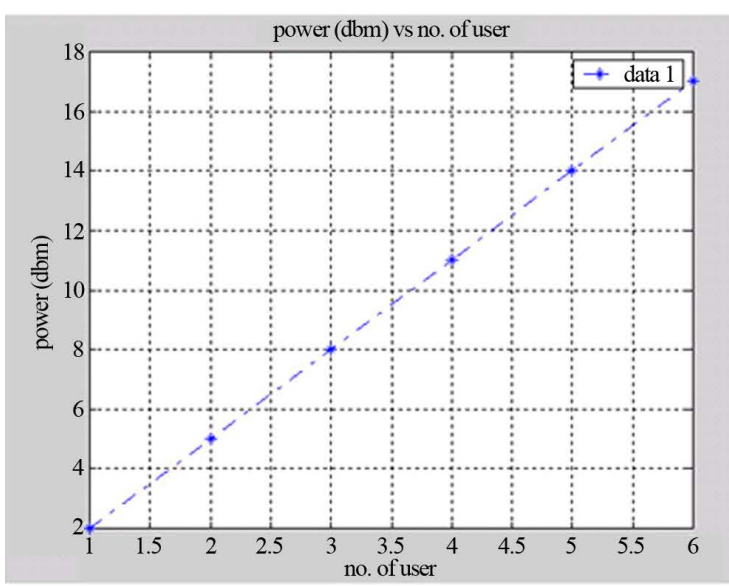

(b)

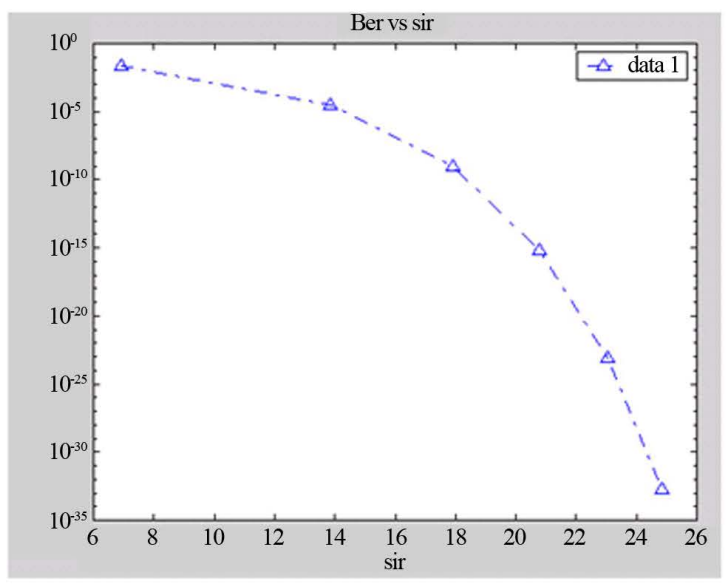

(d)

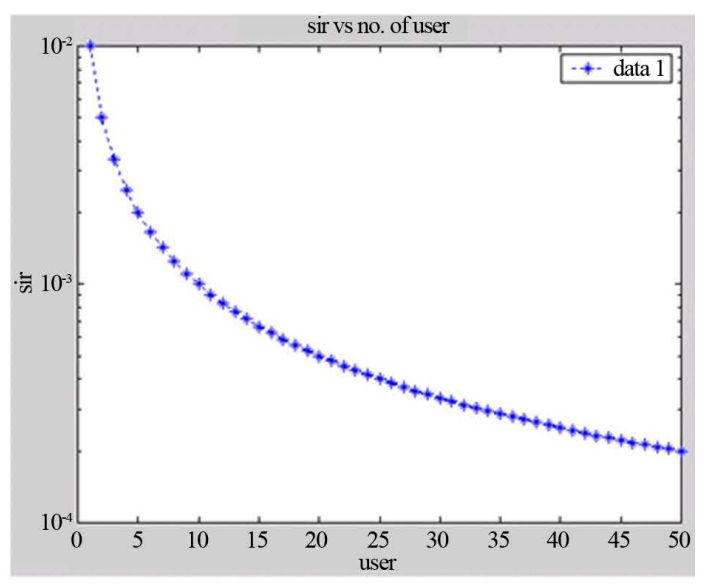

(c)

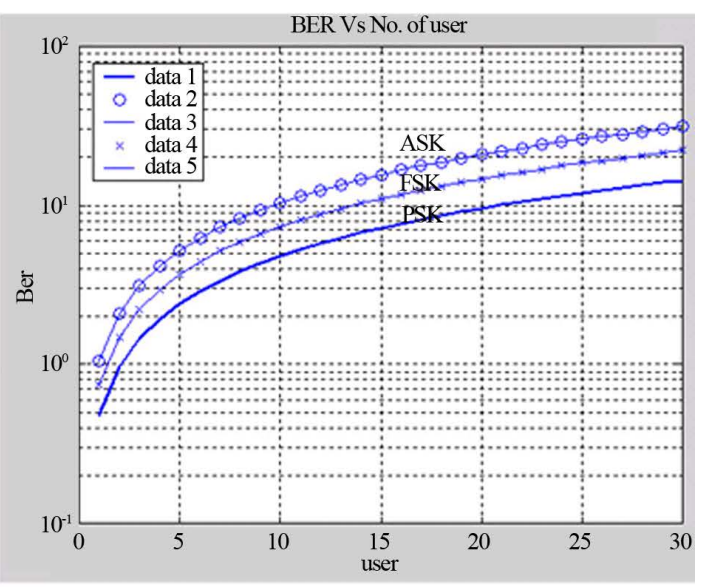

(e)

Figure 6. (a) BER vs. SNR, (b) no. of user vs. power, (c) SIR vs. no. of simultaneous users in a DS-CDMA system, (d) SIR vs. BER and (e) BER vs. no. of simultaneous users in a DS-CDMA system.

of users can be accommodated for PSK compared to ASK and FSK.

In this research, simulation was also carried out to evaluate the output waveforms for a given input data sequences. The waveforms are represented in Figures 7(a)-(d) through Figures 7 (a)-(d). The plot (Figure 7(a)) shows the data bits input to the CDMA encoder. It was found that the input data bits are $[+1,-1,-1,+1,+1,+1$, 


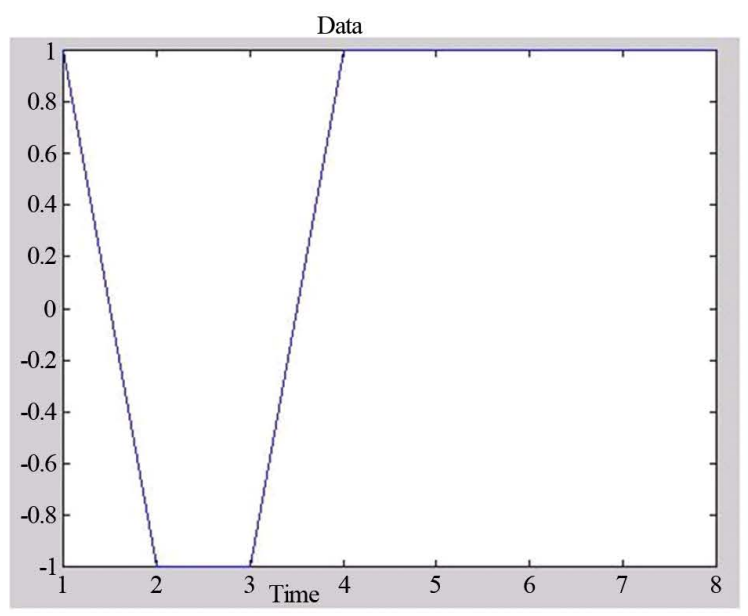

(a)

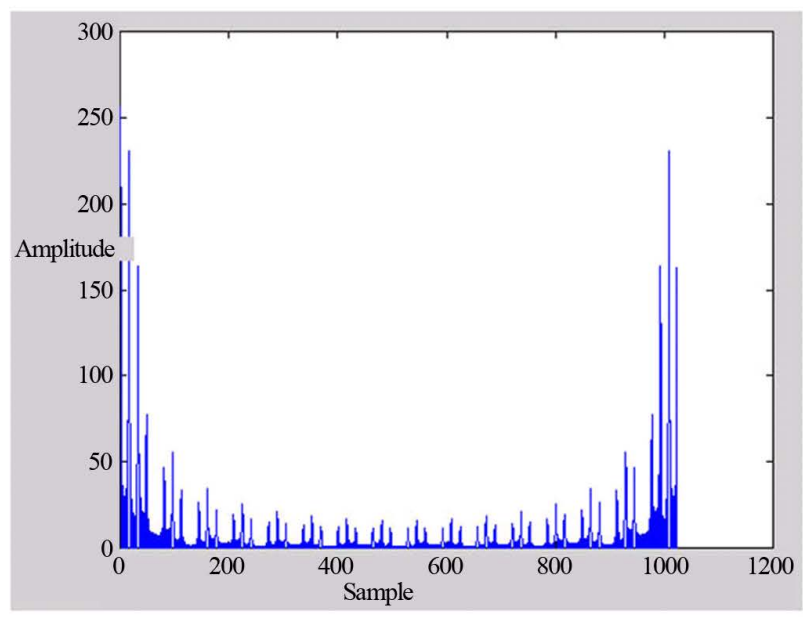

(c)

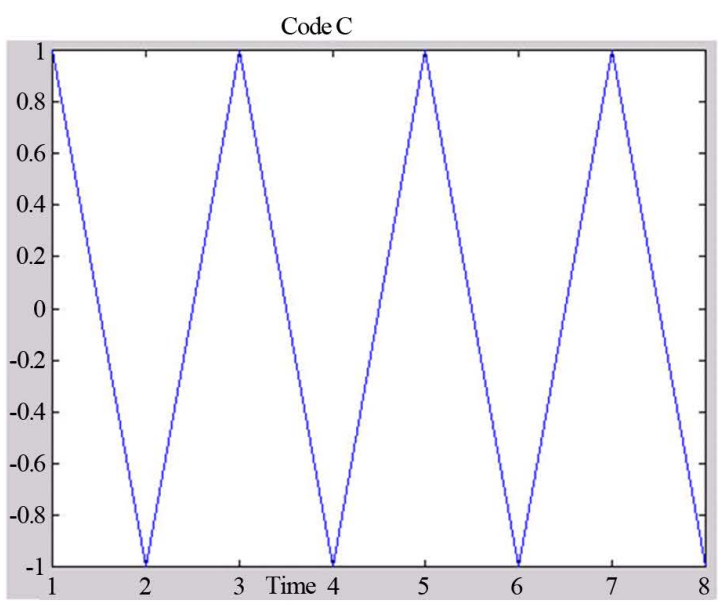

(b)

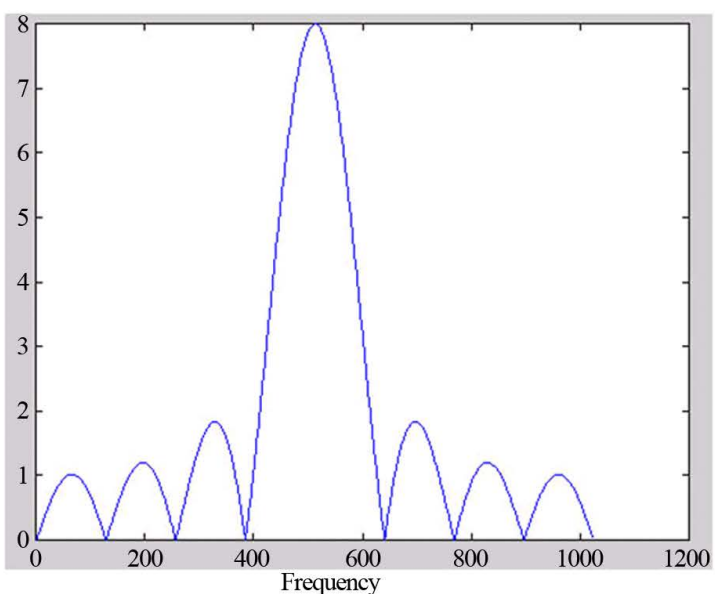

(d)

Figure 7. (a) The data bits input to the CDMA encoder, (b) the code bits sequence, (c) The resultant coding signal and (d) The frequency domain waveform of Spreading signal.

$+1,+1]$. Figure 7 (b) shows the code bits sequence of the CDMA encoder. The code sequence was $[+1,-1,+1$, $-1,+1,-1,+1,-1]$. The spectrum of the input coded signal has been represented in Figure 7(c). The Figure 7(d) represents the frequency domain waveform of spreading signal in the DS-CDMA system. The Figure 8(a) shows the time domain waveform of modulated signal. Figure 8(b) represents the random noise generation. Where the noise power was $10-3 \mathrm{db}, \mathrm{SNR}=10$. Figure 8(c) represents the waveform of modulated signal with noise. Figure 8(d) represents the filtered signal waveform. Filtering was performed to get superior signal quality. Figure 8(e) represents the demodulated waveform. Demodulation was performed to the recover the original signal wave form. Figure 8(f) shows the decoded waveform where we have seen the half portion.

\section{Conclusion}

Using theoretical analyses simulation in MATLAB environment is carried out to extract the numerical results and to draw the possible conclusions for the systems. Considering the acceptable BER is equal to or less than $10^{-3}$ for satisfactory performance of the systems, several conclusions have been made from the numerical results. The possible conclusions are summarized as: 1) For perfect power control in both the cellular and non-cellular DS-CDMA mobile communication systems, the processing gain and the SNR per bit $\mathrm{E}_{\mathrm{b}} / \mathrm{N}_{\mathrm{o}}$ increases, the SINR increases and the BER decreases of the receiver which is an indication of the improvement of the system performance, 2) For perfect power control in both the cellular and non-cellular DS-CDMA mobile communication systems, the number of fading path or signal processing path increases, the SINR decreases and the BER 


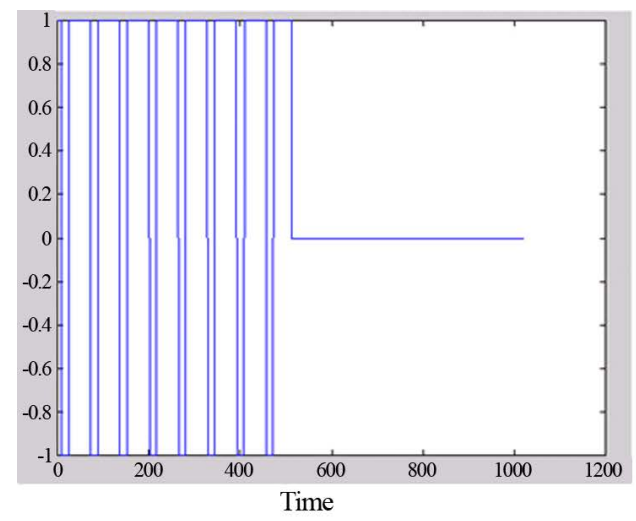

(a)

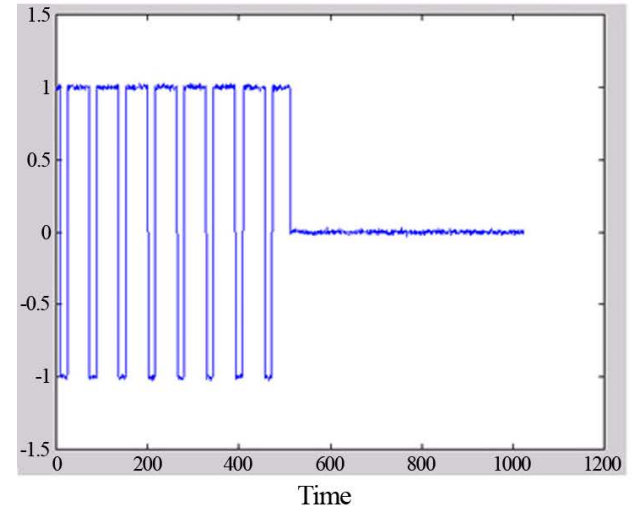

(c)

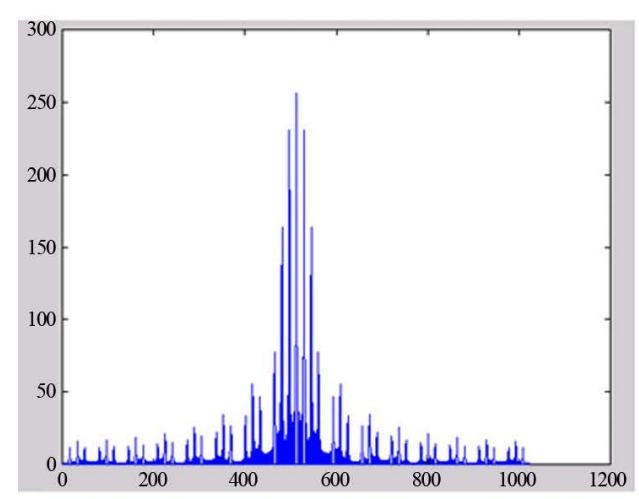

(e)

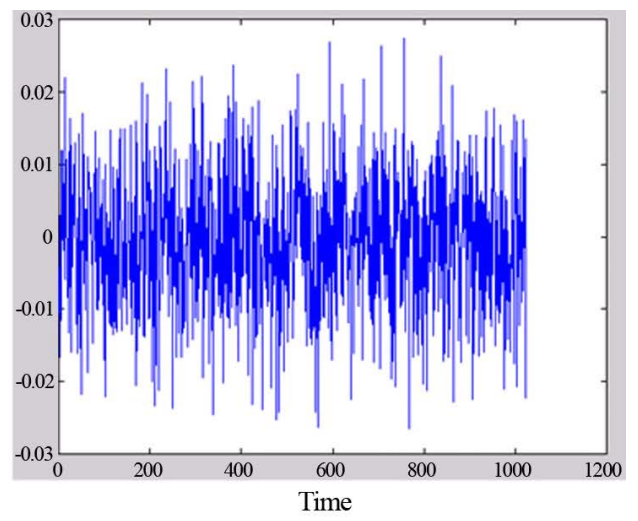

(b)

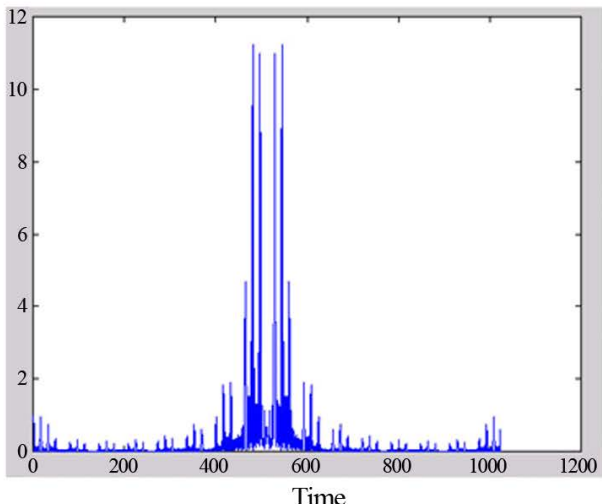

(d)

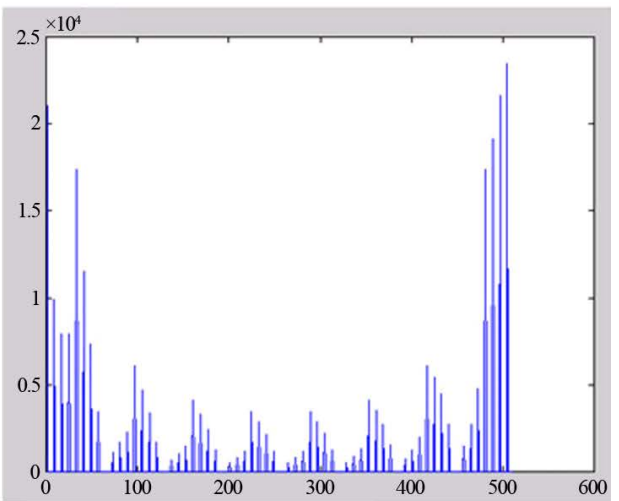

(f)

Figure 8. (a) The modulated waveform, (b) noise generation, (c) added noise with modulated signal, (d) the filtered signal, (e) the demodulated waveform and (f) the decoded waveform.

increases which means the degradation of the system performance, 3) In the perfect power control the performance of cellular and non-cellular DS-CDMA mobile communication systems degrades as the number of interfering cell increases and 4) For both the case of cellular and non-cellular (single cell) DS-CDMA mobile communication systems, the performance is the best for the PPC system and the worst for the absence of power control (APC) system.

\section{Acknowledgements}

We would like to thank all concerned with the APECE department for their all-out effort to support us for completing this research. 


\section{References}

[1] Feher, K. (1995) Wireless Digital Communication: Modulation \& Spread Spectrum Applications. Prentice-Hall Inc., Upper Saddle River.

[2] Rappaport, T.S. (2002) Wireless Communication: Principal and Practice. 2nd Edition, Pearson Education Inc., New York.

[3] Lee, W.C.Y. (2004) Mobile Cellular Telecommunications: Analog and Digital Systems. 2nd Edition, New York.

[4] Faruque, S. (1996) Cellular Mobile System. Engineering, Boston and London.

[5] Lee, Y. (1998) Mobile Communication. Engineering, New York.

[6] Forozan, B.A. (2006) Data Communications and Networking. 3rd Edition, Tata McGraw-Hill, Noida.

[7] Tripathi, N. and Reed, J.H. (2014) Cellular Communication. Wiley-IEEE Press.

[8] Garg, V.K. and Wilkes, J.E. (1996) Wireless and Personal Communication Systems. Prentice-Hall Inc., Upper Saddle River.

[9] Sklar, B. (1988) Mobile Communications Fundamental and Application. Prentice-Hall Inc., Upper Saddle River.

[10] Steele, R. (1999) Mobile Communications. 2nd Edition, Wiley.

[11] Mithal, G.K. (1999) Radio Engineering. Applied Electronics Vol. II. Khpub.

[12] Haykin, S. (2009) Communication Systems. 5th Edition, John Wiley \& Sons, Inc.

[13] Hossain, Md.F. Performance Analysis of DS-CDMA Wireless and Mobile Communication System in a Nakagami-m Frequency Selective Multi-Path Fading Channel. M. Sc Engineering Thesis, Bangladesh University of Engineering and Technology (BUET), Dhaka.

[14] https://en.wikipedia.org/wiki/Rayleigh_fading

[15] Proakis, J.G. (1995) Digital Communications. 3rd Edition, McGraw-Hill Book Co., Singapore, 767-768.

[16] Sklar, B. (1997) Rayleigh Fading Channels in Mobile Digital Communication Systems Part I: Characterization. IEEE Communications Magazine, 35, 90-100. http://dx.doi.org/10.1109/35.601747 